\title{
Front/Rear axle Torque Vectoring Control for Electric Vehicles
}

\author{
David Ruiz Diez \\ Chassis Engineering \\ Jaguar Land Rover \\ United Kingdom \\ Email: drd1807@gmail.com
}

\author{
Davide Tavernini \\ Centre for Automotive Engineering \\ University of Surrey \\ United Kingdom \\ Email: d.tavernini@surrey.ac.uk
}

\author{
Efstathios Siampis \\ Delta Motorsport \\ Silverstone Technology Park \\ United Kingdom \\ Email: stathis@delta-motorsport.com
}

\author{
Efstathios Velenis \\ Advanced Vehicle Engineering Centre \\ Cranfield University \\ United Kingdom \\ Email: e.velenis@cranfield.ac.uk
}

\author{
Edward N. Smith \\ Advanced Vehicle Engineering Centre \\ Cranfield University \\ United Kingdom \\ Email: e.smith@cranfield.ac.uk
}

\author{
Amir Soltani \\ Advanced Vehicle Engineering Centre \\ Cranfield University \\ United Kingdom \\ Email: m.m.soltani@cranfield.ac.uk
}

Vehicles equipped with multiple electric machines allow variable distribution of propulsive and regenerative braking torques between axles or even individual wheels of the car. Left/right torque vectoring (i.e. torque shift between wheels of the same axle) has been treated extensively in the literature, however fewer studies focus on torque shift between the front and rear axles, namely front/rear torque vectoring, a drivetrain topology more suitable for mass production since it reduces complexity and cost. In this paper we propose an online control strategy that can enhance vehicle agility and "fun-to-drive" for such a topology or, if necessary, mitigate oversteer during sub-limit handling conditions. It includes a front/rear torque control allocation strategy is formulated in terms of physical quantities that are directly connected to the vehicle dynamic behaviour like torques and forces, instead of non-physical control signals. Hence, it is possible to easily incorporate the limitations of the electric machines and tyres into the computation of the control action. Aside from the online implementation, this publication includes an offline study to assess the effectiveness of the proposed control allocation strategy, which illustrates the theoretical capability of affecting yaw moment that the front/rear torque vectoring strategy has for a given set of vehicle and road conditions, and considering physical limitations of the tyres and actuators. The development of the complete strategy is presented together with results from Hardware-in-the-Loop (HiL) simulations, using a high fidelity vehicle model and covering various use cases.

\section{Introduction}

The emerging technology of the electric vehicle has provided not only an answer to the global need for a reduction in transportation sector emissions but also new possibilities for improving the handling characteristics of the vehicle. Specifically, in the case of electric vehicles equipped with two or more electric machines that drive/brake different axles or wheels, techniques such as the torque vectoring method can be applied to enhance handling characteristics, stability and fun-to-drive aspects of the vehicle. Torque Vectoring (TV), the method of controlling both the direction and magnitude of torque on different axles or across axles in order to influence the dynamics of the vehicle, has been successfully applied on conventional drivelines using expensive and complex differentials. However, on a vehicle equipped with electric machines, torque vectoring can be readily applied with less compromises, by virtue of their high frequency torque delivery and accuracy of response.

In this study we consider the development and validation of a front/rear torque vectoring controller for an electric vehicle that can independently control the driving/braking torque of each axle (one electric machine per axle), where torque in evenly distributed between left and right via open differentials.

Most torque vectoring systems reported in the literature fo- 
cus on the generation of a corrective yaw moment by the vehicle in order to follow a specified yaw rate demand. In a vehicle with two electric motors on the front and/or rear axle, a left/right torque vectoring solution is also possible [1-4]. Such topology can produce the corrective yaw moment by wheel-independent torque requests that result in a differential of longitudinal forces between the left and right sides of the vehicle. However, a significant yaw moment contribution arises from the manipulation of tyre lateral forces induced by the change in the longitudinal forces, through tyre force coupling effects. This aspect can be fully exploited in a front/rear TV strategy. Despite the fact that such a method has less potential to change the dynamics of the vehicle when compared to left/right torque vectoring, it can still improve the vehicle handling behaviour. For example Piyabongkarn [5] has shown how if torque is transferred from the front to the rear wheels of the vehicle, then a more oversteering behaviour relative to the current dynamic handling balance is induced. This idea has been implemented by $\mathrm{He}$ et al. in [6], where a simple torque allocation strategy is proposed: whenever the yaw rate deviation from the yaw rate reference indicates an understeer vehicle behaviour, the torque is shifted towards the rear to induce oversteer and vice versa, with no further consideration of the dynamics of the vehicle. Another example of a front/rear torque vectoring solution, this time using a novel central differential with a small electric motor for torque modulation, was the focus of research by Wheals et. al. [7-9]. Here a bicycle model is used to relate the steady-state yaw rate with the front and rear lateral stiffnesses of the tyres (assumed equal left/right). A control allocation algorithm finds the combination of the front/rear tyre stiffness that is able to most closely track the steady-state yaw rate target. Then, employing a nonlinear tyre model, the required lateral stiffnesses are translated to longitudinal slip ratio demands and finally electric machine torque requests on the front and rear axle. While results show that the dynamics of the car can be considerably modified and improved, a disadvantage of this strategy is that the calculations are made in terms of stiffness of the tyres and yaw rate, rather than in terms of required forces and moments, which makes it difficult to consider the physical limits of the system. In [10], a front/rear torque vectoring strategy tries to equalise the level of lateral force saturation of the front and rear axle. To estimate saturation levels, a handling vehicle model with non-linear tyre model is used to consider the interdependency between lateral and longitudinal forces. The torque allocation strategy on the other hand, is a simple PI controller minimising level of saturation between front and rear axle, pursuing a vehicle behaviour as linear as possible. This approach presents some advantages over close loop yaw control methods, such as naturally adapting to the level of grip of the road without actually estimating the road-tyre adhesion coefficient.

A front/rear torque vectoring strategy for stabilization of the vehicle at the limits of handling using a Model Predictive Control (MPC) strategy can be found in [11]. Here a combined longitudinal and lateral dynamics control is applied to the problem of overspeeding through a turn, with simulation results showing that path deviation can be minimised by appropriate use of the front and rear axle electric motors.

In this study we present a front/rear torque vectoring control strategy that achieves a yaw gain feel more linear and consistent than a car with no active systems, in all conditions, such as variations in longitudinal acceleration. It can be used also to modify the handling balance character of the car, enhancing its agility and "fun-to-drive" aspect or, alternatively, mitigating its oversteer.

The proposed TV control system is not intended to replace stability control but to complement it. As it is demonstrated in the following sections, the control authority is limited compared to four wheel differential braking. It aims to actively modify handling characteristics during combined acceleration/braking and cornering using the vehicle topology of one motor driving each axle, commonly used for allwheel-drive electric vehicles.

Our study confirms front/rear torque vectoring as an effective approach to enhance vehicle handling. The main contribution is a front/rear TV control strategy designed to generate a corrective yaw moment by a control allocation algorithm formulated in a novel way. The problem is expressed in quantities that can be directly connected to physical limitations of the drivetrain components including electric machines torque and power limits and tyre adhesion limits. In this way, a longitudinal acceleration/yaw moment envelope will be shown, which allows to assess the effectiveness and limitations of the approach off-line. Moreover, by using online enforcement of limits, actuator saturation is more likely to be avoided whilst simultaneously distributing the available torque most efficiently.

After a brief introduction of the integrated control architecture, this publication presents the different functions of the Controller:

- The computation of the yaw rate reference.

- The online estimation strategy (based on an Unscented Kalman Filter).

- The computation of longitudinal forces and corrective yaw moment to be applied to the vehicle (High Level Controller).

- The Control Allocation strategy.

Then, this publication presents an off-line analysis of the front/rear TV layout and the proposed Control Allocation Strategy to illustrate its capabilities and limitations . Finally, the complete solution is then tested in a Hardware-in-theLoop (HiL) simulation environment in order to assess realtime capability and effectiveness of the proposed solution on a high fidelity vehicle model. The results from multiple manoeuvres show the effectiveness of the proposed torque vectoring strategy in modifying the yaw response of the car in different scenarios.

\section{IVCS: Integrated Vehicle Control Structure}

In this section, we describe the control system architecture and key components that the Control Allocation (CA) 


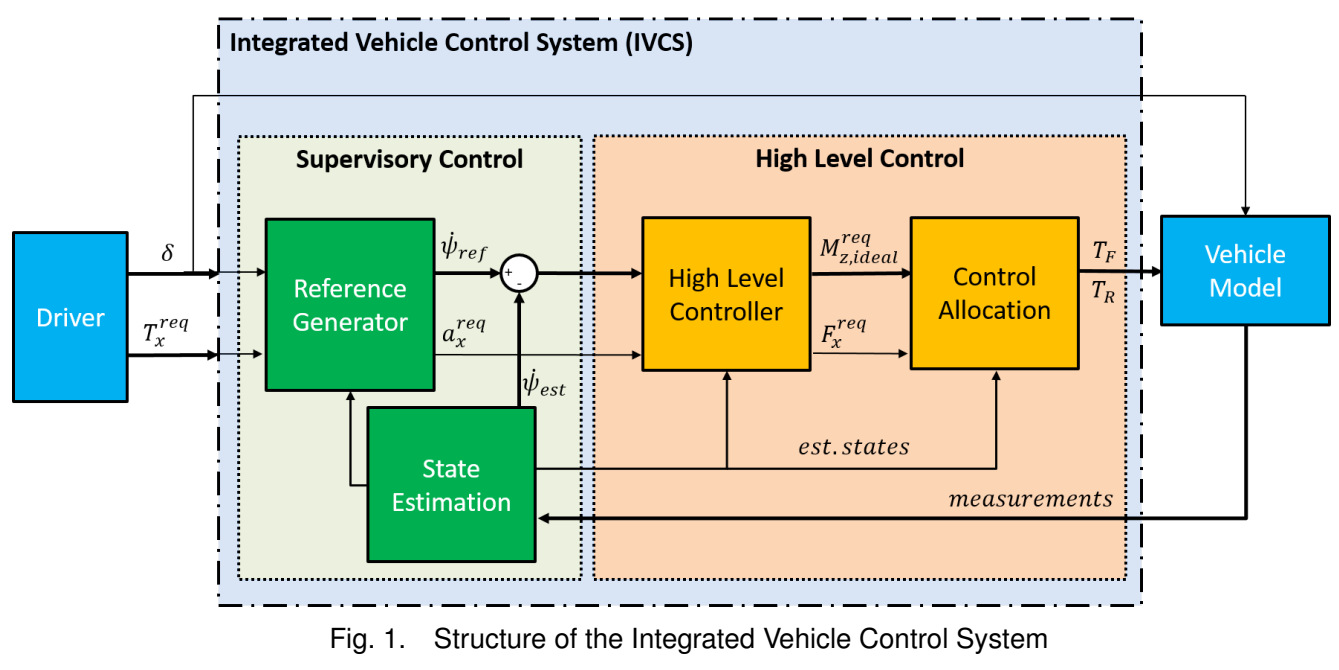

sits within. We have adopted a centralised Integrated Vehicle Control Structure (IVCS) [12] that allows multiple Vehicle Dynamics Control (VDC) systems to be coordinated together, an approach also found in the literature for control of over-actuated vehicles [13,14]. The structure (Fig. $1)$, reduces complexity and eases the model-based design process from Model-in-the-Loop (MiL), suitable for desktop computing using low and high fidelity plant models to Hardware-in-the-Loop testing (HiL) to test for real-time implementation before installation on test vehicles. The IVCS is a hierarchical structure composed of:

\section{Supervisory Control}

This unit includes State Estimation which provides the variables of interest based on the available measurements and the Reference Generator that produces the longitudinal acceleration demand from the driver intention (i.e. accelerator pedal) and the yaw rate reference.

\section{High Level Control}

This unit, is composed of High Level Controller and Control Allocation blocks. The former takes the demand in terms of vehicle motion (i.e. acceleration, yaw rate) and converts them into forces and moments acting on the vehicle; acceleration is transformed into a total longitudinal force demand and yaw rate error is supplied to a PID controller to generate a yaw moment demand, as explained in section 3.1. The CA (section 5) calculates the torque request for the actuators (front and rear electric motors in this case) in order to deliver the yaw moment demand from the High level controller.

In the following sections, each component of the IVCS is treated in more detail, with a specific emphasis on the Control Allocation scheme, which is the core contribution of this work.

\section{Supervisory Control}

The Supervisory Control layer (figure 2) is concerned with specifying motion objectives in terms of yaw rate and forward acceleration, which requires estimation of certain states.

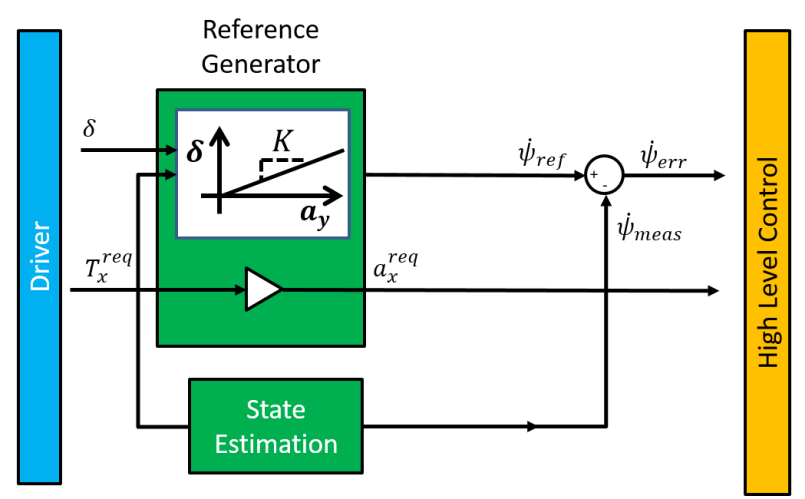

Fig. 2. Supervisory Control level of IVCS

\subsection{Reference Generator}

Motion objectives such as yaw rate reference and a longitudinal acceleration reference are calculated by the Reference Generator. The latter is generated by conversion of the longitudinal torque demand, $T_{x}^{r e q}$, from the pedal map into longitudinal acceleration demand, $a_{x}^{\text {req }}$. The yaw rate reference is generated by consideration of the steady-state solution of the classical linear-tyre bicycle vehicle model [15-17]:

$$
\dot{\psi}_{r e f}=\frac{V}{\left(\ell_{f}+\ell_{r}\right)+V^{2} K_{U}} \delta
$$

where $\dot{\psi}_{\text {ref }}$ is the yaw rate reference, $V$ is the vehicle velocity, $\ell_{r}$ and $\ell_{r}$ are the distances from the centre of mass (CG) to the front and rear axles respectively. $K_{U}$ is the understeer gradient, defined as:

$$
K_{U}=\frac{m\left(\ell_{r} C_{r}-\ell_{f} C_{f}\right)}{\left(\ell_{f}+\ell_{r}\right) C_{f} C_{r}}
$$

where $m$ is the vehicle mass and $C_{f}$ and $C_{r}$ are the front and rear tyre cornering stiffnesses of the linear tyre model respectively. The reference yaw rate is saturated with the 


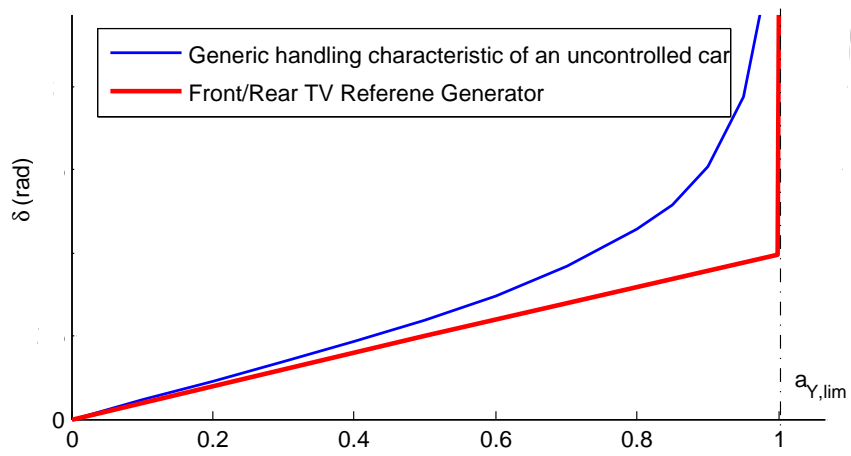

Fig. 3. Wheel steer angle plotted against steady state lateral acceleration, for a generic uncontrolled car, and for the proposed Front/Rear TV controller. The gradient of the curves is the understeer gradient of the vehicle as defined in 2 . The magnitude of the $y$-axis (steer angle) depends on the vehicle speed.

road-tyre adhesion coefficient $\mu$ so that the the value of the reference generated remain within feasible values, using the expression below:

$$
\dot{\psi}_{\text {ref }}<=\frac{a_{Y, l i m}}{V}=\mu g \frac{1}{V}
$$

The estimation of $\mu$ is not within the scope of this publication. Although its value has been set to 1 , the online controller implementation can adjust to different input values of $\mu$ if a robust estimation of that state was available, adjusting its performance to different road conditions.

When the steer angle at the front wheels $\delta$ is plotted against lateral acceleration $a_{y}$, the gradient of the resulting curve is the understeer gradient. Figure 3 shows the typical steady state handling behaviour of an uncontrolled car (without Torque Vectoring, i.e. fixed torque distribution), which exhibits a highly non-linear behaviour towards the limit lateral acceleration $a_{y, \text { lim }}$ due to tyre force coupling. For simplicity, a completely linear yaw target up to the adhesion limit has been used, as the focus of this publication is the Control Allocation. More sophisticated yaw rate reference shaping can be found in $[2,3]$.

\subsection{Estimation}

In this section, an Unscented Kalman Filter (UKF) formulation is presented to estimate the variables of interest. The development and test results of the UKF has been published in [18], where it has proven good results to estimate the sideslip angle of the vehicle with much lesser computational effort than an Extended Kalman Filter (EFK) .

Assuming that no additional sensors to the standard set found in most vehicles are used, the available measurements of interest are the longitudinal and lateral acceleration of the vehicle, along with yaw rate from the Inertial Measurement Unit (IMU), the rotational speeds on the wheels from the four

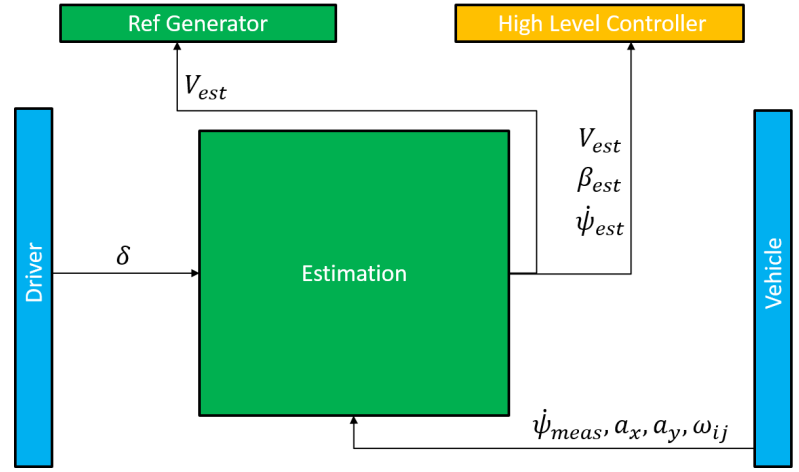

Fig. 4. Estimation block

wheel speed sensors and the steering wheel angle from the wheel angle sensor. Note at this point that we assume that no measurements of the torque on the wheels are available.

The UKF uses a four-wheel nonlinear vehicle model [18] descrived by the system:

$$
\begin{aligned}
& \dot{x}=f(x, u)+w, \\
& y=g(x, u)+v,
\end{aligned}
$$

where $x, u$ and $y$ are the state, input and output vectors respectively, and $w, v$ the process and measurement noises with covariance matrices $Q$ and $R$ respectively. The vectors $x, u$ and $y$ are defined as:

$$
x=\left[\begin{array}{c}
V \\
\beta \\
\psi
\end{array}\right], \quad u=\left[\begin{array}{c}
\delta \\
\omega_{i j}
\end{array}\right], \quad y=\left[\begin{array}{c}
a_{x} \\
a_{y} \\
\dot{\psi}
\end{array}\right]
$$

where $\beta$ is the vehicle sideslip and $\omega_{i j}$ the wheel angular speeds on each corner. Using the wheel speeds as inputs to the estimation model instead of the wheel torques, we obtain a compact form for the estimator that allows for faster computation and avoids relying on wheel torque measurements. The measurement noise covariance matrix $R$ is set according to information about sensor noise levels while the process noise covariance matrix $Q$, which represents parameter uncertainties and unmodeled dynamics, was found through simulation studies, with the values used for both matrices presented in Table 1. The resulting $\mathrm{Q}$ and $\mathrm{R}$ matrices are captures in equation (6) and (7) . Finally, the sampling time is set to $6 \mathrm{~ms}$, while for the prediction step 10 steps of the Euler integration were used, the latter giving results comparable to a Runge-Kutta scheme at a lower computational cost.

$$
Q=\left[\begin{array}{ccc}
1 \times 10^{-3} & 0 & 0 \\
0 & 1 \times 10^{-3} & 0 \\
0 & 0 & 1 \times 10^{-2}
\end{array}\right]
$$


Table 1. Noise mean and variance values per signal.

\begin{tabular}{ccc}
\hline & Mean & Variance \\
\hline$a_{x}$ & 0 & $1 \times 10^{-1}$ \\
$a_{y}$ & 0 & $1 \times 10^{-2}$ \\
$\dot{\psi}$ & 0 & $1 \times 10^{-6}$ \\
$\delta$ & 0 & $1 \times 10^{-6}$ \\
$\omega_{i j}$ & 0 & $7 \times 10^{-2}$
\end{tabular}

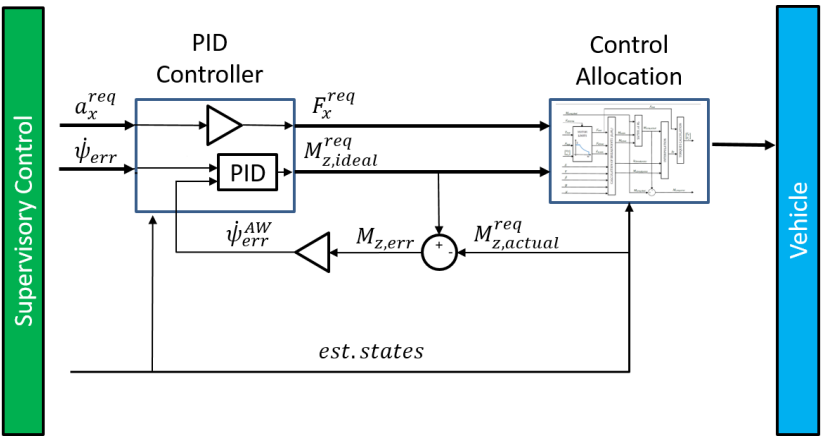

Fig. 5. High level control

$$
R=\left[\begin{array}{ccc}
1 \times 10^{-1} & 0 & 0 \\
0 & 1 \times 10^{-2} & 0 \\
0 & 0 & 1 \times 10^{-6}
\end{array}\right]
$$

\section{High Level Controller}

The High Level Controller block consists of a gainscheduled PID controller comprising anti-windup and reset features. An "ideal" form PID, with the proportional gain multiplying the sum of all of the actions is used, including a filter on the derivative term [19]. In discrete form, the formulation is as follows:

$$
\begin{aligned}
M_{z, \text { rea }}^{\text {ideal }}(z) & =P\left[\left(1+I\left(\frac{T_{s}}{z-1}\right)+D\left(\frac{N}{1+N\left(\frac{T_{s}}{z-1}\right)}\right)\right) \dot{\psi}_{\text {err }}\right. \\
& \left.-I\left(\frac{T_{s}}{z-1}\right) \dot{\Psi}_{\text {err }}^{A W}\right],
\end{aligned}
$$

where: $M_{z, \text { req }}^{\text {ideal }}$ is the ideal requested yaw moment, not considering actuator saturation or friction limits; $\dot{\psi}_{\text {err }}$ is the yaw-rate error; $P, I, D, N$ are the proportional, integral, derivative gains and filter coefficient respectively; $T_{S}$ is the sampling time. The yaw rate correction for anti-wind up is defined as:

$$
\dot{\psi}_{\text {err }}^{A W}=K^{A W}\left(M_{z, \text { req }}^{\text {ideal }}-M_{z, \text { req }}^{\text {actual }}\right),
$$

where $K^{A W}$ is the anti-windup gain. Integral anti-windup was a necessary addition to the PID functionality, to account for friction limits and motor torque and power limits that restrict the yaw moment that the vehicle is capable of achieving. In our solution, shown in Figure 5, a correction in the yaw-rate error, $\dot{\psi}_{\text {err }}^{A W}$, to be subtracted from the yaw-rate error fed to the integral term is calculated by taking the difference $\left(M_{z, r e q}^{e r r}\right)$ between the ideal yaw moment request, $M_{z, r e q}^{\text {ideal }}$ (output by the PID) and actual yaw moment request, $M_{z, \text { req }}^{\text {actual }}$ (output from the Control Allocation block, considering friction and motor limits) multiplied by the gain $K^{A W}$.

Gain scheduling by lookup table is implemented for the
$\mathrm{P}, \mathrm{I}$ and $\mathrm{N}$ gains, which are tuned as a function of tyre-road adhesion coefficient. The I gain is also tuned according to the understeer gradient target and the longitudinal force demand. The $\mathrm{N}$ gain is set dependent on the $\mathrm{D}$ gain.

\section{Control Allocation}

The Control Allocation calculates the motor torques on each axle in order to simultaneously deliver the longitudinal total force request $F_{x, \text { req }}^{\text {tot }}$ and the vehicle yaw moment demand $M_{z, r e q}$ from the PID controller. A parameter $k_{f}$ is defined as:

$$
k_{f}=\frac{F_{x f}}{F_{x}^{\mathrm{tot}}}=\frac{F_{x f}}{F_{x f}+F_{x r}} .
$$

This is, $k_{f}$ is the longitudinal force at the front axle over the overall longitudinal force applied to the vehicle and represents the front/rear torque distribution that is the output of the allocation algorithm. When $0 \leq k_{f} \leq 1$, both longitudinal forces are applied in the same direction (acceleration or braking). The application of a longitudinal force distribution outside these limits results in longitudinal forces being applied in opposite directions, i.e. the front axle under drive torque with the rear braking and vice versa. Whenever the longitudinal acceleration demand and the yaw rate demand cannot be met simultaneously, the allocation algorithm will give priority to the former to respect the driver's longitudinal request.

\subsection{Control Allocation structure}

A schematic of the Control Allocation is shown in Fig. 6 and consists of:

1. The Motor Limits block, which computes the maximum torques applicable to each axle considering the physical torque and power limits of the electric machines. The speed of each axle is considered as the average between left and right wheels. This results in a maximun torque on the front and rear axles, $T_{x f, \text { max }}$ and $T_{x r \text { max }}$ respectively, which are used to saturate the driver's longitudinal demand $F_{x, \text { req }}^{\text {ideal }}$ into $F_{x t o t}$.

2. The "Calculation of feasible yaw moments" block is the core calculation of the algorithm and obtains values of 


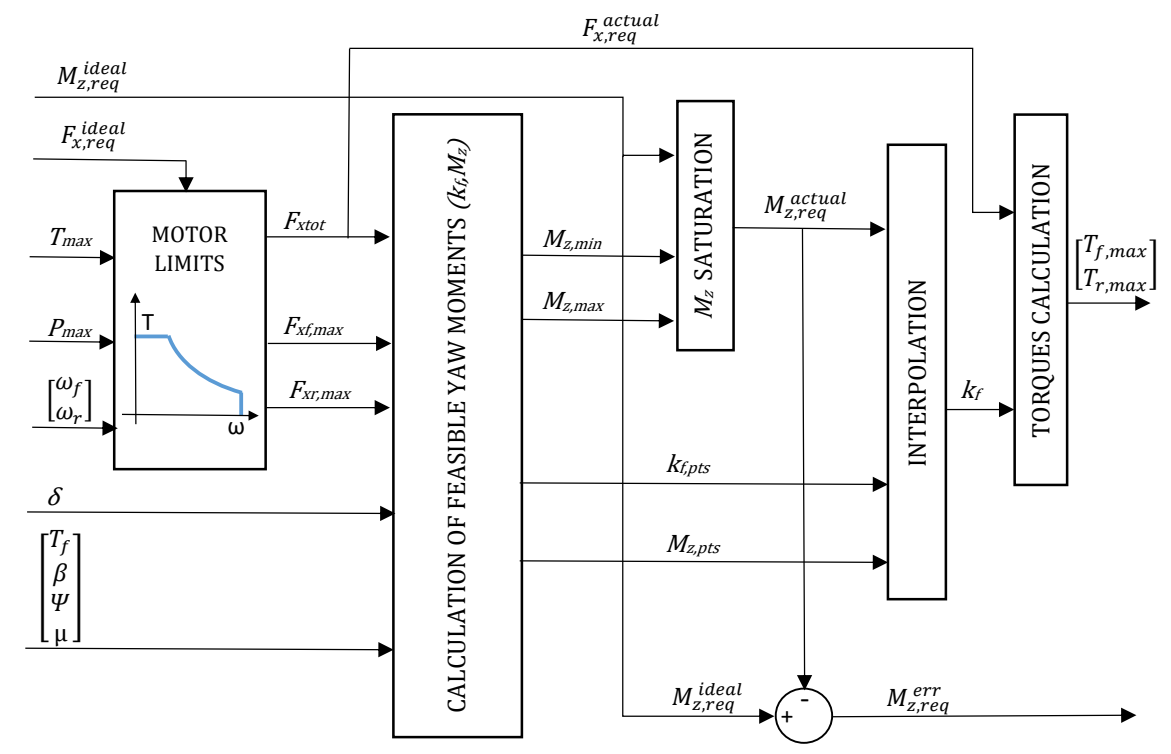

Fig. 6. Schematic of the Allocation Algorithm

achievable vehicle yaw moments $\left(M_{z, p t s}\right)$ corresponding to different longitudinal force distributions $\left(k_{f, p t s}\right)$. These are calculated from the single-track vehicle model with a nonlinear tyre model (presented in section 5.2), with the method described in section 5.3. The final $\mathrm{kf}$ that corresponds to the requested Mz,req,actual, is obtained by interpolating between Mz,pts and kf,pts. Mz,pts will include, as a minimum, the maximum and minimum yaw moment that can be generated, $M_{z, \min }$ and $M_{z, \max }$, respectively.

3. The $M_{z}$ Saturation block, in which the yaw moment request $M_{z, r e q}^{\text {ideal }}$ from the controller constrained by $M_{z, \text { min }}$ and $M_{z, \max }$, producing $M_{z, \text { req }}^{\text {actual }}$.

4. The Interpolation block obtains the $k_{f}$ distribution that provides $M_{z, \text { req }}^{\text {actual }}$ by interpolation of $M_{z, p t s}$ and $k_{f, p t s}$. Providing that the accuracy provided by the number of points used for the interpolation is enough, the Control Allocation will provide the best longitudinal force distribution $k_{f}$, according to the non-linear single-track model.

5. The Torques calculation block delivers the output torque demands for each axle. The longitudinal force request on each axle are obtained simply by multiplying $k_{f}$ (or $1-k_{f}$ for the rear case) by the saturated longitudinal force request $F_{x, r e q}^{\text {actual }}$, and then converted into the torque requests on each axle $T_{f, r e q}$ and $T_{r, \text { req }}$.

6. The error, $M_{z, \text { req }}^{\text {err }}$, between $M_{z, \text { req }}^{\text {actual }}$ and requested $M_{z, \text { rea }}^{\text {ideal }}$ (i.e. desired and theoretically achievable yaw moments) is used for anti-windup control action by the PID controller.

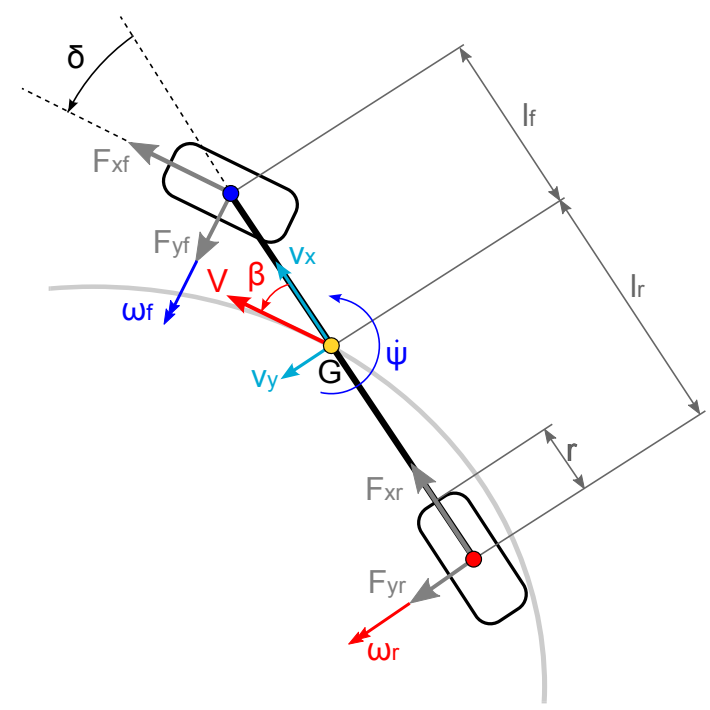

Fig. 7. Single track vehicle model employed for available yaw moment calculation.

\subsection{Vehicle modeling for control strategy computations}

In order to calculate the yaw moment generated from a combination of front and rear longitudinal forces, we use a single-track vehicle model (Fig. 7) with 5 degrees of freedom: longitudinal velocity $v_{x}$, lateral valocity $v_{y}$, yaw rate $\psi$ and angular velocities of the front and rear wheels, $\omega_{f}$ and $\omega_{r}$ respectively. Pitch and roll are neglected. The lateral forces $F_{y f}$ and $F_{y r}$ in Fig. 7 correspond to the sum of the lateral forces of both wheels of the respective axle. Same applies to the longitudinal forces on each axle, $F_{x f}$ and $F_{x r}$. The vehicle body equations of motion are given by:

$$
m\left(v_{x}-v_{y} \dot{\psi}\right)=F_{x r}+F_{x f} \cos \delta-F_{y f} \sin \delta
$$




$$
m\left(\dot{v}_{y}+v_{x} \dot{\psi}\right)=F_{y r}+F_{y f} \cos \delta+F_{x f} \sin \delta,
$$

$$
M_{z}=\left(F_{y f} \cos \delta+F_{x f} \sin \delta\right) l_{f}-F_{y r} l_{r}
$$

where $m$ is the total mass of the vehicle, $M_{z}$ is the vehicle yaw moment, $l_{r}$ and $l_{f}$ are distances of the centre of gravity $G$ from rear and front axle respectively. Wheels dynamics equations are as follows:

$$
J_{w} \dot{\omega}_{f}=T_{f}-F_{x f} r, \quad J_{w} \dot{\omega}_{r}=T_{r}-F_{x r} r
$$

with axle inertia, $J_{w}$, and effective rolling radius, $r$, equal for front and rear. Front and rear sideslip angles can be computed from:

$$
\alpha_{f}=\delta-\frac{v_{y}+\dot{\psi} l_{f}}{v_{x}}, \quad \alpha_{r}=-\frac{v_{y}-\dot{\psi} l_{r}}{v_{x}} .
$$

Theoretical lateral slip (15), in its simplified form (practical longitudinal slip effect neglected) becomes:

$$
\sigma_{y f} \approx \tan \alpha_{f}, \quad \sigma_{y r} \approx \tan \alpha_{r} .
$$

Longitudinal theoretical slips can be computed as:

$$
\sigma_{x f}=\frac{\omega_{f} r-v_{x}}{\omega_{f} r}, \quad \sigma_{x r}=\frac{\omega_{r} r-v_{x}}{\omega_{r} r} .
$$

The coupling between longitudinal and lateral forces is accounted using the similarity method described in [20]. From this, the equivalent slip is calculated:

$$
\sigma_{f}=\sqrt{\sigma_{x f}^{2}+\sigma_{y f}^{2}}, \quad \sigma_{r}=\sqrt{\sigma_{x r}^{2}+\sigma_{y r}^{2}} .
$$

As a result of this, the tyre adhesion utilisation for combined longitudinal and lateral efforts is defined using a simplified version of the Pacejka Magic Formula [20]:

$$
\mu_{i}=D \sin \left(C \arctan \left(\sigma_{i} B\right)\right)
$$

Normalized tyre forces are then defined:

$$
\begin{gathered}
\mu_{x i}=\frac{\sigma_{x i}}{\sigma_{i}} D \sin \left(C \arctan \left(B \sigma_{i}\right)\right), \\
\mu_{y i}=\frac{\sigma_{y i}}{\sigma_{i}} D \sin \left(C \arctan \left(B \sigma_{i}\right)\right)
\end{gathered}
$$

with i=f,r, and $B, C, D$ Pacejka coefficients used to define both lateral and longitudinal characteristics. Front and real longitudinal forces:

$$
F_{x i}=\mu_{x i} F_{z i}, \quad F_{y i}=\mu_{y i} F_{z i},
$$

with $F_{z i}$ front and rear vertical loads on the tyres. Vertical loads can be simply obtained as the static load on each axle plus or minus the longitudinal weight transfer due to the longitudinal acceleration:

$$
\begin{gathered}
F_{z f}=\frac{l_{r} m g-h m \dot{v}_{x}}{m g}, \\
F_{z r}=\frac{l_{f} m g+h m \dot{v}_{x}}{m g} .
\end{gathered}
$$

where $h$ is the height of the vehicle's centre of gravity.

\subsection{Calculation of feasible yaw moments}

In this section we now explain in detail the calculation of feasible yaw moments that represents the core of the CA.

Inputs required are: the overall longitudinal force request $F_{x, \text { req }}^{\text {actual }}$, the maximum longitudinal forces that each motor can deliver on each axle, $F_{x f, \max }$ and $F_{x r, \max }$, and a set of vehicle states. Steering angle $\delta$ and yaw rate $\dot{\psi}$ are measured and vector velocity $V$ and sidelip angle $\beta$ come from estimation (section 3.2).

The crucial information we require are the maximum and minimum attainable yaw moments, $M_{z, \max }$ and $M_{z, \min }$. Following the ISO sign convention for a left hand turn, applying $M_{z, \max }$ (i.e. greatest value of positive yaw moment) induces the maximum possible shift towards oversteer (reduction in understeer relative to instantaneous handling balance) produced by the minimum longitudinal force distribution $k_{f, \min }$, which sends the maximum possible torque to the rear axle. Conversely, $M_{z, \min }$ (i.e. greatest value of negative yaw moment) induces the highest possible shift towards understeer (reduction in oversteer relative to instantaneous handling balance) produced by the maximum distribution $k_{f, \max }$. Further explanation is given in section 6 .

$k_{f, \min }$ and $k_{f, \max }$ are restriced within the interval $[0,1]$ so that the torque on each axle is always applied in the same direction to ensure good energy efficiency, reduce tyre wear and avoid damage to driveline components. $k_{f, \min }$ and $k_{f, \max }$ may be limited further due to tyre-road adhesion limits or electric machines limits. $M_{z, \max }$ and $M_{z, \min }$ constitute the theoretical limits of front/rear TV capability. The smallest number of interpolation points required are the pairs $\left(k_{f, \min }\right.$, $\left.M_{z, \max }\right)$ and $\left(k_{f, \max }, M_{z, \min }\right)$. Additionally, we calculate the yaw moments corresponding to several intermediate values of $k_{f}$, such that $k_{f, \min }<k_{f}<k_{f, \max }$, and use interpolation to obtain $M_{z}\left(k_{f}\right)$. The number of intermediate $k_{f}$ depends on 
the required accuracy.

The parameter $k_{f}$ is incorporated in the equations of motion of the nonlinear vehicle presented in section 5.2 (11-13) as appropriate to obtain equations (24-26), respectively.

$$
\begin{aligned}
& m\left(\dot{v}_{x}-v_{y} \dot{\psi}\right)= \\
& =k_{f} F_{x, r e q}^{\text {actual }}+\left(1-k_{f}\right) F_{x, r e q}^{\text {actual }} \cos \delta-F_{y f} \sin \delta, \\
& m\left(\dot{v}_{y}+v_{x} \dot{\psi}\right)=F_{y r}+F_{y f} \cos \delta+k_{f} F_{x, r e q}^{\text {actual }} \sin \delta, \\
& M_{z}=\left(F_{y f} \cos \delta+k_{f} F_{x, r e q}^{\text {actual }} \sin \delta\right) l_{f}-F_{y r} l_{r}
\end{aligned}
$$

Equations (24) and (26) represent the two targets the CA is tasked with providing: longitudinal torque request (driver-demand) and yaw moment request (desired handling response from reference generator). Equation (25) would only be needed if side-slip control was required. A singletrack model is used since torques and longitudinal forces on each axle are equal left and right. Equations (15-22) capture the coupling between longitudinal and lateral tyre forces which is the phenomenon that front/rear TV controller hinges on.

The resulting system of equations is solvable considering $F_{x, r e q}^{\text {actual }}, k_{f}$ and the states of the vehicle as inputs. However, due to the nonlinearities in the tyre model equations (20) \& (21), no direct algebraic solution can be found. Therefore this problem has been solved using an iterative numerical method summarised in the following steps:

1. Calculate tyre normal forces. They are obtained from (23).

2. Calculate lateral tyre slips. In order to do so, first the lateral speed of the car at its centre of gravity is estimated using the following geometric equation:

$$
v_{y}=V \tan \beta
$$

where $V$ and $\beta$ are estimated as explanied in section 3.2. Then the lateral tyre slips at the front and rear axles, $\alpha_{s} f$ and $\alpha_{y r}$ can be obtained from (15) and (16).

3. Obtain physical limits of the motors and tyres. The tyre model proposed in equation (19) will provide a curve of combined longitudinal and lateral tyre adhesion utilisation that will peak at a certain combined tyre slip. These tyre slips, namely $\sigma_{f, \max }$ and $\sigma_{r, \max }$, are the maximum combined slips that can be attained on each axle without exceeding the adhesion limits. These can lead to the maximum longitudinal tyre slips on each axle if equation (18) is rewritten as follows:

$$
\sigma_{x i, \max }=\sqrt{\sigma_{i, \max }^{2}-\sigma_{y i}^{2}}
$$

Finally, the maximum normalised longitudinal forces that can applied on each axle not to exceed the adhesion limit, from substitution in equation (19), are:

$$
\mu_{x i, \max }=\frac{\sigma_{x i, \max }}{\sigma_{i, \max }} D \sin \left(C \arctan \left(B \sigma_{i, \max }\right)\right),
$$

On the other hand, from equation (14), the electric motors on each axle can deliver a maximum longitudinal torque defined:

$$
F_{x i, \max }=\frac{T_{i, \max }-J_{w} \dot{\omega}_{i}}{r},
$$

4. Allocation of axle longitudinal forces. For the longitudinal request $F_{x, r e q}^{\text {tot }}$ and a given longitudinal force distribution $k_{f}$, the longitudinal forces requested on each axle are intially set to:

$$
F_{x f}=k_{f} F_{x, r e q}^{\text {actual }}, \quad F_{x r}=\left(1-k_{f}\right) F_{x, \text { req }}^{\text {actual }}
$$

However, if these longitudinal forces are larger than the adhesion limit from (30) or than the tyre forces than the motors can deliver (equation 31), they are saturated. In that case, $k_{f}$ would be recalculated with the corrected (saturated) longitudinal forces.

5. Calculation of axle lateral forces. Here is where the iterative process takes places, in order to obtain the real longitudinal slip ratios. For each iteration, a guess of longitudinal slip is made for each axle; lateral slips are always known from step 1 and hence the equivalent overall slip on each axle can be obtained from (18). To finish each iterative step, the tyre longitudinal and lateral forces are calculated from (20) and (21). The longitudinal slip guesses are updated and the same process repeated until the value of longitudinal force calculated matches that from step (4) within a certain tolerance.

6. Calculate the generated maximum and minimum yaw moment, from (13).

\subsection{Practical considerations of the Control}

An alternative torque splitting strategy is used instead that maximises adhesion utilisation: $k_{f, a l t}$ is proportional to the normal load on each axle, calculated as per equation (23). 
In some circumstances, it is not worth using the TV strategy because the effect achievable is non existant or too small (e.g. straight line driving, very low vehicle speeds, very small longitudinal forces or whenever the lateral slip ratios are very small). This will be better justified with the results from (6) Therefore, whenever the TV range, defined as the difference between $M_{z, \max }$ and $M_{z, \min }$, is less than a certain threshold, the system is deactivated. An alternative torque splitting strategy is used instead that maximises adhesion utilisation: $k_{f, \text { alt }}$ is proportional to the normal load on each axle, calculated as per equation (23).

$$
k_{f, a l t}=F_{z f} /\left(F_{z f}+F_{z r}\right)
$$

A smooth transition between the two modes was provided in the controller to prevent harsh output torques. This transition function blends-in the front/rear TV torque distribution $k_{f}$ and blends-out the alternative torque distribution $k_{f, \text { alt }}$ in a linear manner with respect to the TV range $\left(M_{z, \max }\right.$ - $\left.M_{z, \min }\right)$ as the later increases, an vice versa when it decreases below a certain threshold.

\section{Offline study of Front/Rear TV capabilities}

This section uses the same equations and approach as the Control Allocation to develop an offline study to illustrate the capabilities of the control strategy, although it is not required as part of the online implementation. A great advantage of a formulation based on forces and moments acting on the vehcle is that it allows us to perform an anlaysis of the effectivness of the front/rear TV, its capabilities and limits for the available actuators and the driving conditions off-line. Graphic results can be generated using the method described in section 5.3 for various longitudinal requests and longitudinal force distibutions $k_{f}$. Figure 8 shows the results considering the bicycle model paramaterised for a lightweight sports car for a fixed set of vehicle states: high adhesion conditions $(\mu=1), \beta=3 \mathrm{deg}, v=100 \mathrm{~km} / \mathrm{h}, \dot{\psi}=$ $20 \mathrm{deg} / \mathrm{s}$ and $\delta$ set to the Ackermann steer angle. Results are shown for a left-hand turn, using the ISO sign convention. Vehicle yaw moments equal to zero exhibit neutral steer behaviour, positive yaw moments push the car towards oversteer and negative yaw moments produce understeer behaviour.

The results confirm that for a certain level of acceleration, higher values of $k_{f}$ will shift more torque towards the front axle, decreasing the lateral capability of that axle and hence will always induce a more understeering behaviour, reducing oversteer. On the other hand, smaller values of $k_{f}$ will always induce oversteer or work towards reducing the degree of understeer.

The yaw moment envelope that can be obtained is enclosed by the curves associated with adhesion limits in which either front or rear axle would exceed the tyre grip capabilities if a longitudinal force any higher were applied. Curves showing different levels of maximum motor torque are also shown.
It can be observed that the curve corresponding to a 50:50 front/rear fixed distribution $\left(k_{f}=0.5\right)$ is inclined, since the model accounts for longitudinal weight transfer, resulting in more oversteer during braking (positive yaw moments) and more understeer (negative yaw moments) under positive acceleration.

We can draw some further interesting conclusions from these results: during steady-state cornering or under small longitudinal accelerations/decelerations, the longitudinal slip/forces are too small to alter lateral forces sufficiently to be able to influence the vehicle path trajectory significantly. However, if $k_{f}$ values higher than 1 or lower than 0 are permitted, the front/rear TV capabilities can be extended for low and medium longitudinal accelerations. As already explained, this means that the longitudinal forces on each axle are applied in opposite directions, improving handling performance at the expense of energy consumption and powertain and tyre longevity.

During less aggressive cornering (low sideslip), the yaw moment that can be generated is significantly reduced, due to lower tyre lateral slips and forces. This does not mean, however, that the front/rear TV has reduced capability in low adhesion conditions, since the yaw moment required to adjust the vehicle trajectory is also lower.

Figure 8 can also be used as a visual description of how to determine the breakpoints for selecting the $k_{f}$ front/rear distribution parameter described in section 5.1. Consider, for example, a longitudinal acceleration of $a_{x}=4 \mathrm{~m} / \mathrm{s}^{2}$; this will define a vertical line where the points for interpolation will lie on. To find the maximum yaw moment possible at this acceleration, we first select the point that lies on the $k_{f}=0$ dashed line: $\simeq 900 \mathrm{Nm}$. Now assuming the motors are limited to $1400 \mathrm{Nm}$ (red curve), the maximum yaw moment can still be delivered and remains $\simeq 900 \mathrm{Nm}$. To find the minimum yaw moment, we attempt to select the point that lies on the $k_{f}=1$ dashed line. We see, however, that this is not possible, since it would lie outside the limits of adhesion. Thus we take as the minimum yaw moment $\simeq-3400 \mathrm{Nm}$ by a distribution of $k_{f} \simeq 0.85$. Therefore the values used for interpolation, at this longitudinal acceleration, are $\left(k_{f, \min }, M_{z, \max }\right)=(0,900 \mathrm{Nm})$ and $\left(k_{f, \text { max }}, M_{z, \text { min }}\right)=(0.85,-3400 N m)$. The $k_{f}$ needed to most closely achieve the desired yaw moment demand is computed by linear interpolation between these points.

\section{Hardware in the Loop implementation and results}

The proposed TV control strategy was implemented in a dSPACE MicroAutoBox Rapid Control Prototyping platform on Cranfield University Hardware-in-the-Loop (HiL) facility [12] for fine tuning and validation. The plant represents a battery electric vehicle (BEV) which runs in real-time on a dSPACE Midsize Simulation Platform and it is based on high fidelity vehicle dynamics model from the software CarMaker plus a model of the electric parts such as batteries, Permanent Magnet Synchronous Motor (PMSM) motors and low-level motor controllers. The signal 


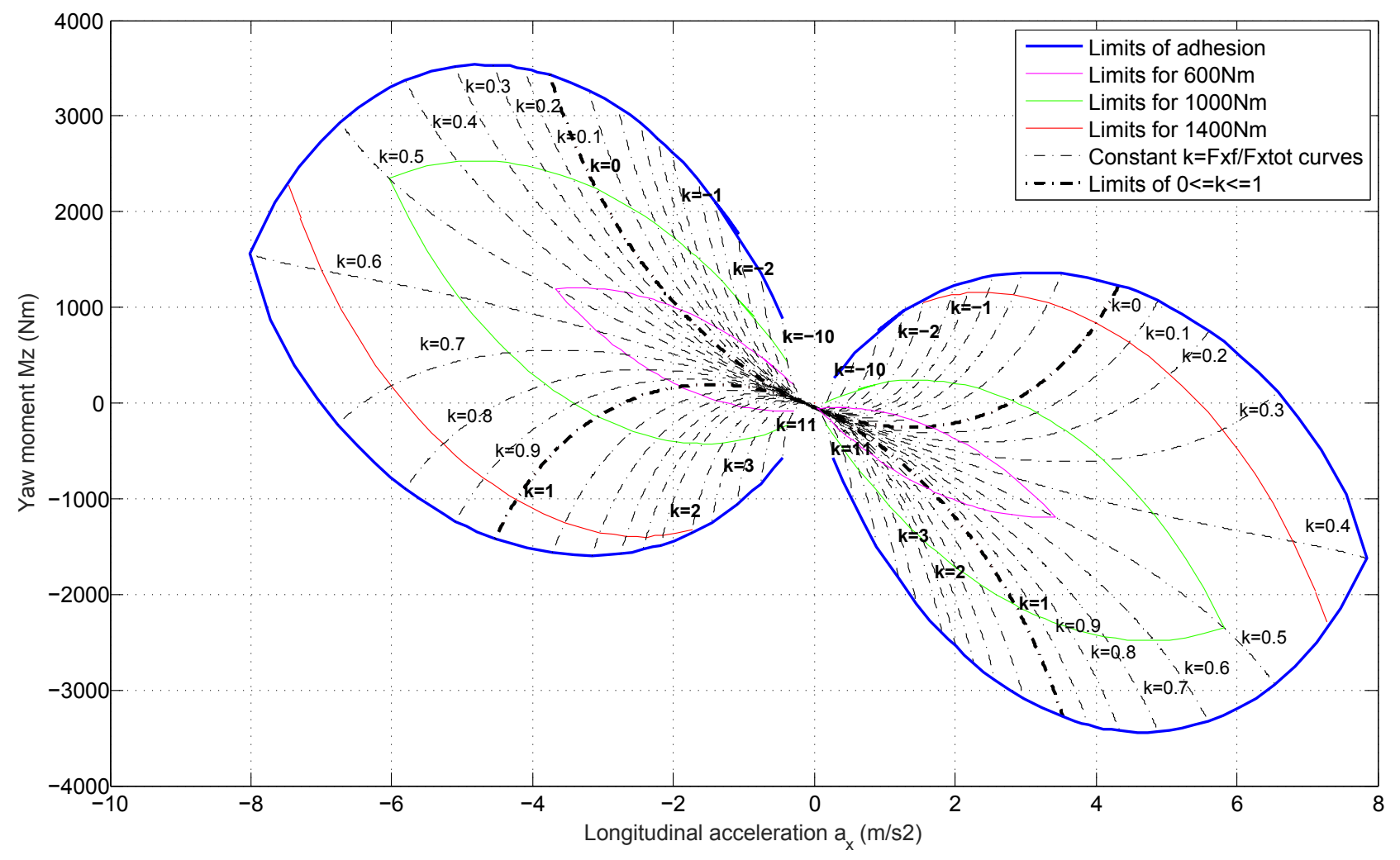

Fig. 8. Front to rear TV map, high adhesion $(\mu=1)$ and high vehicle sideslip ( $\beta=3 \mathrm{deg}), \nu=10 \mathrm{~km} / \mathrm{h}, \psi=20 \mathrm{deg} / \mathrm{s}$ and $\delta=1.7 \mathrm{deg}$

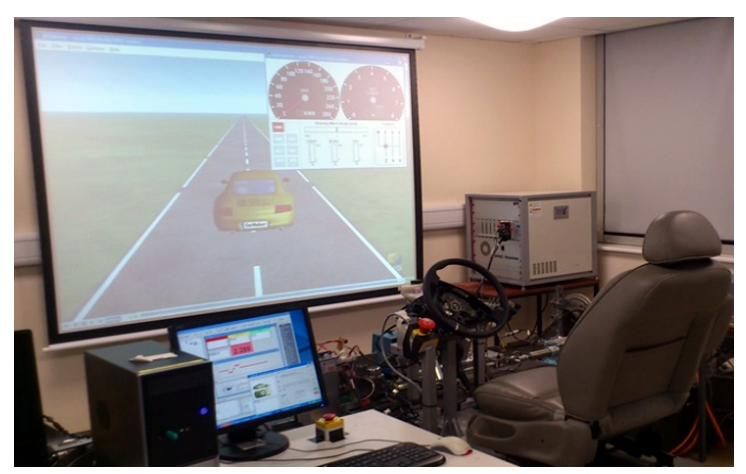

Fig. 9. HiL Setup: driver set-up with visualisation

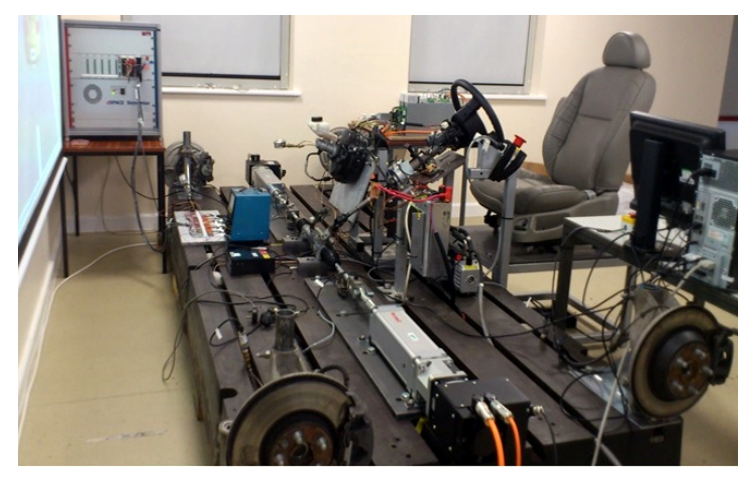

Fig. 10. HiL Setup: EPAS with by-wire braking system to/from the HiL subsystems, including the vehicle simulator, the prototyping ECU, the sensors and the actuators, are communicating via a CAN bus, so the effect of associated latencies and time delays on control system performance are considered. Noise has been introduced artificially to the input signals as random oscillatory signals to replicate the conditions of the sensor signals in a real car. The vehicle model corresponds to a lightweight sports battery electric vehicle.

The manoeuvres utilised for the controller development can be classified as step-steer and constant steer - in either case, a constant longitudinal acceleration has been provided throughout the entire manoeuvre-. One step steer manoeuvre and one constant steer manoeuvre have selected to be shown in this section, with different understeer targets, which are presented below. All manoeuvres were performed in dry tarmac with a tyre-road adhesion coefficient of 1 .

\subsection{Step-steer manoeuvre with constant acceleration}

The car is subject to a constant longitudinal acceleration of $0.4 \mathrm{~g}$ and, at $40 \mathrm{~km} / \mathrm{h}$, a step-steer of $50 \mathrm{deg}$ at the steering wheel is applied up until $85 \mathrm{~km} / \mathrm{h}$, when the steering is returned to the straight position. The same manoeuvre is repeated for three different understeer targets: $K_{U}=0.0$ $\mathrm{deg} / \mathrm{g}$ (which represents oversteer when compared to the uncontrolled car, which exhibits mild understeer), $K_{U}=-0.5$ 

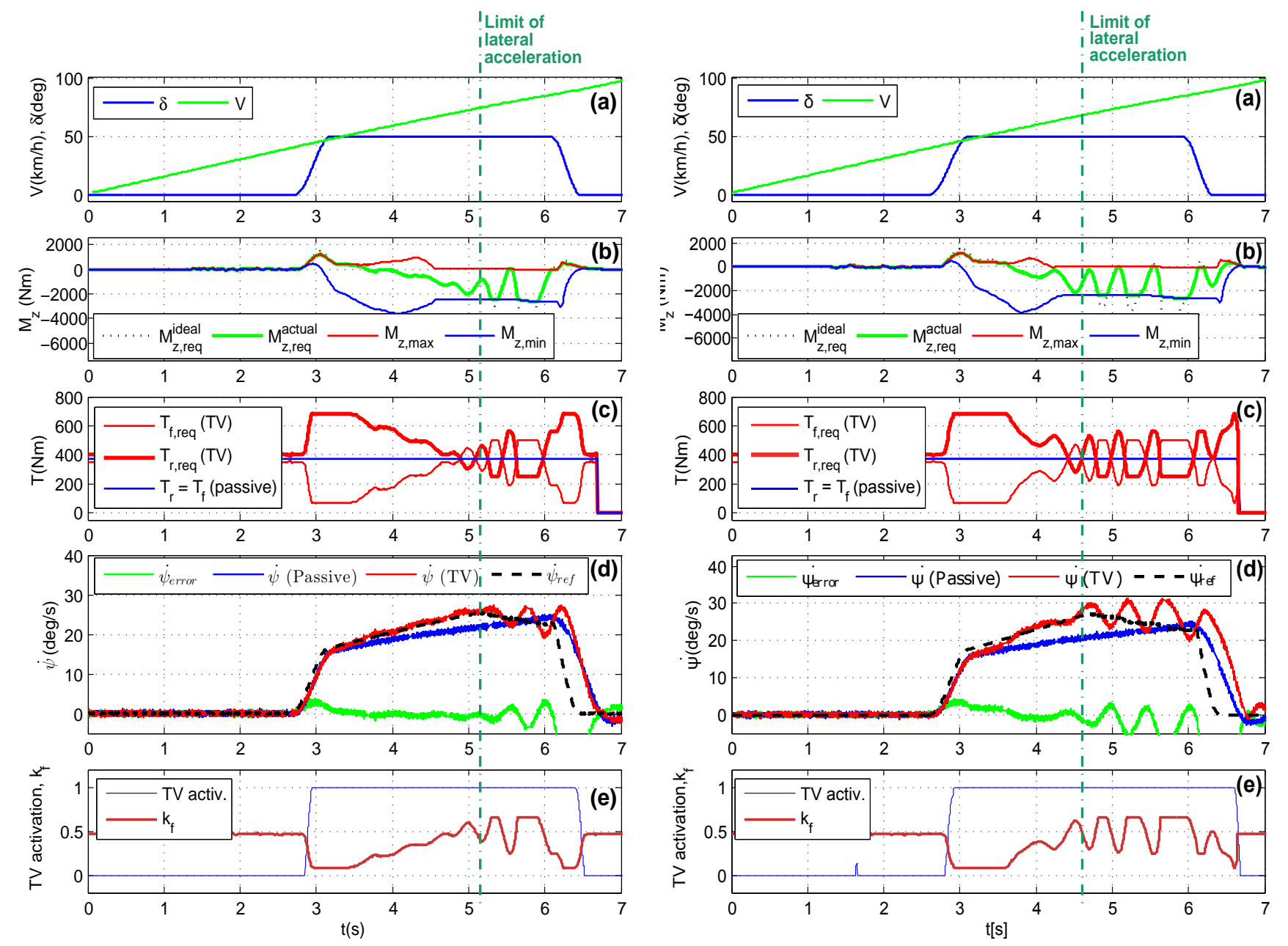

Fig. 11. Constant acceleration step steer manoeuvre, with understeer gradient target $K_{U}=0.0 \mathrm{deg} / \mathrm{g}$ : (a) Steering wheel angle and vehicle speed, (b) Calculated yaw moments in the Allocation Algorithm, (c) Axle torque requests, (d) Measured yaw rates versus yaw rate reference (f) TV transitioning (activation) factor and front/rear torque distribution $k_{f}$

deg/g (a target that implies higher oversteer) and $K_{U}=1.0$ $\mathrm{deg} / \mathrm{g}$ (more understeery than the uncontrolled car). All three cases were benchmarked against the uncontrolled (non TV) car, operating with 50:50 front/rear fixed torque distribution. Figure 11 shows the results for the manoeuvre effectuated when the yaw rate reference is set to an understeer gradient $K_{U}=0.0 \mathrm{deg} / \mathrm{g}$. The TV controller starts to control the torque sent to the front and rear actuators from $\mathrm{t}=2.9 \mathrm{~s}$. At that point, the difference between $M_{z, \max }$ and $M_{z, \min }$ (shown in Figure 11(b)) is big enough to trigger the front/rear TV control, which progressively comes into play as the TV transitioning factor - see Figure 11(e) - changes from 0 (TV not active) to 1 (TV fully modulating the torque between front/rear axles). Initially after applying the step steer, the controller shifts torque to the rear in great proportion to induce oversteer and soon is able to track the yaw rate reference and stabilise the car on that trajectory (Figure 11(d)),

Fig. 12. Constant acceleration step steer manoeuvre, with understeer gradient target $K_{U}=-0.5 \mathrm{deg} / \mathrm{g}$ : (a) Steering wheel angle and vehicle speed, (b) Calculated yaw moments in the Allocation Algorithm, (c) Axle torque requests, (d) Measured yaw rates versus yaw rate reference (f) TV transitioning (activation) factor and front/rear torque distribution $k_{f}$

which is followed closely by modulating the axle torques (as seen in Figure 11(c) and also Figure 11(e), the latter showing the front/rear torque distribution parameter $k_{f}$ ). During the first instant of TV activation, the yaw moment request from the High Level controller $M_{z, \text { real }}^{\text {ide }}$ is saturated at the maximum permitted value (see Figure 11 (b)). Subsequently, the request is satisfied within the available range $\left(M_{z, \max }-M_{z, \min }\right)$. The lateral acceleration increases over the course of the manoeuvre and the range of available yaw moments also increases as a result to a maximum at around $t=4.3 \mathrm{~s}$, to then start decreasing. This is due to the fact that we are getting closer to the limits of adhesion of the tyres, according to the non-linear tyre model employed, and not as much longitudinal force can be shifted to one of the axles without spinning the tyres of that axle or inducing an unstable sideslip angle. At $\mathrm{t}=5.2 \mathrm{~s}$, the lateral acceleration reaches $1.0 \mathrm{~g}$ the yaw reference stops increasing linearly, as this triggers a theoretical 

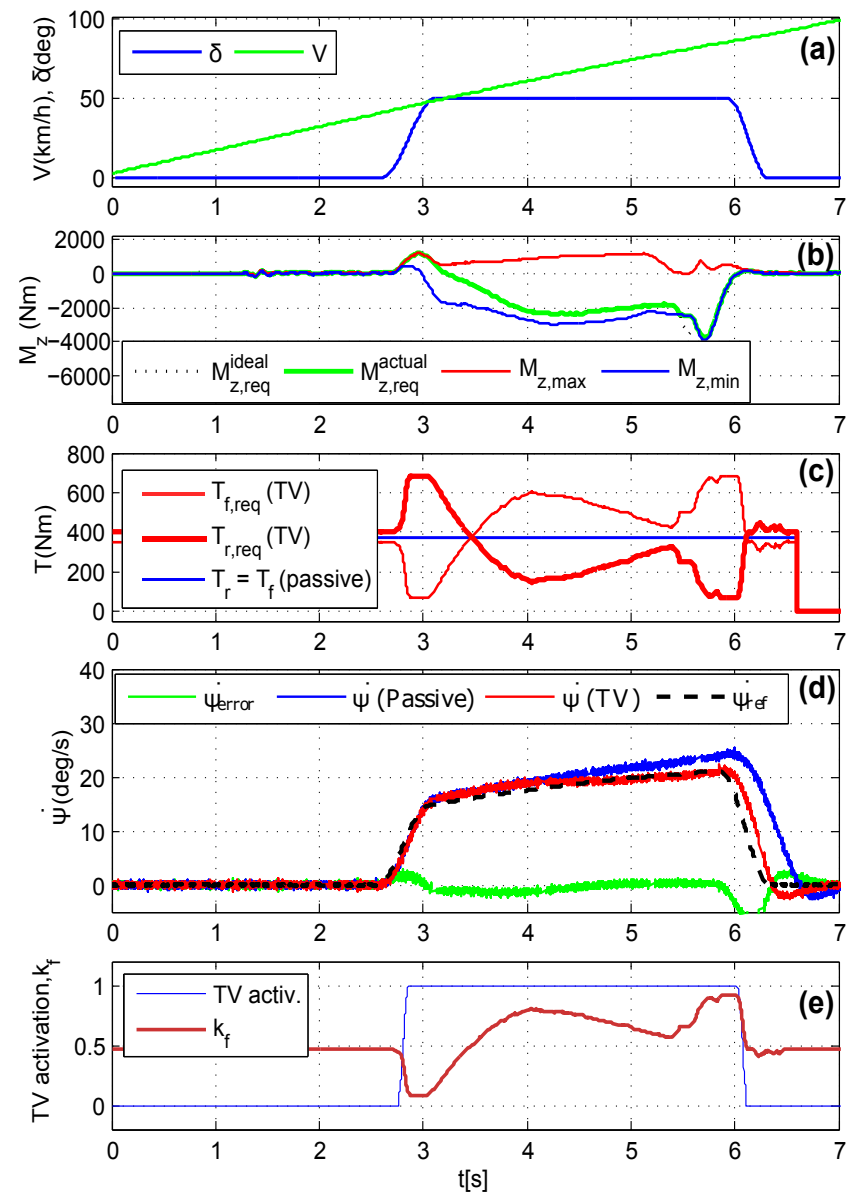

Fig. 13. Constant acceleration step steer manoeuvre, with understeer gradient target $K_{U}=1.0 \mathrm{deg} / \mathrm{g}$ : (a) Steering wheel angle and vehicle speed, (b) Calculated yaw moments in the Allocation Algorithm, (c) Axle torque requests, (d) Measured yaw rates versus yaw rate reference (f) TV transitioning (activation) factor and front/rear torque distribution $k_{f}$

limit of adhesion after which the yaw rate reference is not calculated based on a linear yaw gain anymore, according to the Yaw Reference Generator described in section 3.1. Under this conditions, the TV controller is not able to control the path adequately and oscillates. However, the controller is only intended to control the car's yaw behaviour in sub-limit handling conditions, as the stability control will take over to correct the trajectory of the car at the limit of adhesion. No stability control was simulated in the manoeuvres shown.

Figure 12 shows the same manoeuvre with a higher oversteer target of $K_{U}=-0.5 \mathrm{deg} / \mathrm{g}$. This will lead to higher yaw rates and the maximum lateral acceleration to be reached sooner. When the step steer is applied, the controller tries to induce a higher level of understeer compared to the previous manoeuvre by shifting the maximum allowed torque to the rear axle (Figure 12(b-c)). It is not until around $t=3.8 \mathrm{~s}$ that the yaw rate reference is achieved and closely followed (Fig- ure 12(d)), after which point the controller achieves good tracking. After $\mathrm{t}=4.6 \mathrm{~s}, 1.0 \mathrm{~g}$ of lateral acceleration is reached, which changes the yaw rate reference, entering the region of limit handling.

Lastly, Figure 13 replicates the manoeuvre with $K_{U}=1.0$ $\mathrm{deg} / \mathrm{g}$, which implies an understeer behaviour higher than the exhibited by the uncontrolled car. Although the controller is mainly designed to pursue agility and fun to drive aspect in a car, understeer target were also tested to prove the effectiveness of the proposed solution if a target for overtseer mitigation was applied. Initially, when the step steer is applied, the measured yaw rate lags the reference (see Figure 13(d)) and the controller tries to compensate by introducing a positive yaw moment (Figure 13(b)) that shifts the torque to the rear (Figure 13(c)). As the transient settles, understeer must be induced to follow the reference, and hence the controller changes the torque bias to the front in order to apply a negative yaw moment, after which the trajectory is nicely followed modulating the torque bias (see $k_{f}$ in Figure 13(b)). The step steer is removed at around $t=6.0 \mathrm{~s}$ as previously, but in this case, this happens before the car reaches $1.0 \mathrm{~g}$ of lateral acceleration. As the front wheels are returned to a straight position, the transitioning factor (Figure 13(e)) shifts from 1 to 0 , to progressively deactivate the torque vectoring and move to the alternative front/rear torque distribution, proportional to normal load on each axle to maximise traction as described in section 5.2

The limits of $k_{f}=\left[k_{f, \min }, k_{f, \max }\right]$ vary during the manoeuvre due to the consideration of the limits of the tyre adhesion and the electric motors. All manoeuvres have a constant longitudinal force request which can not be delivered fully by one of the electric motors on its own, therefore initially the distribution factor will be limited to $k_{f}=[0.09,0.91]$ rather than $k_{f}=[0,1]$. Additionally, as the lateral acceleration increases and the vehicle approaches the limits of adhesion, the limits will narrow down further; this starts happening from approximately 4 seconds into the manoeuvers.

The effect of the three different understeer targets on the trajectory, over the passive car, is shown in Figure 14. Once TV is activated, the lateral dynamics of the car are significantly modified by just shifting the driving torque between the front and rear axle. A scaled drawing of the car is given within the Figure to provide a visual comparison in the trajectories, as well as indicators on the trajectory every second.

\subsection{Constant steer with constant acceleration}

The car now starts from standstill with a constant acceleration of $0.4 \mathrm{~g}$, and the steering angle is set to $80 \mathrm{deg}$ at the steering wheel during the whole manoeuvre, until limit handling is reached. Again, the results for this manoeuvre will be shown for different understeer targets $\left(K_{U}=0.0 \mathrm{deg} / \mathrm{g}\right.$ and $K_{U}=1.0 \mathrm{deg} / \mathrm{g}$ in this case) and compared against the uncontrolled vehicle.

Figure 15 shows the manoeuvre when $K_{U}$ is set to $0 \mathrm{deg} / \mathrm{g}$. For this manoeuvre, the target is very close to the passive vehicle. As the speed and lateral acceleration in- 


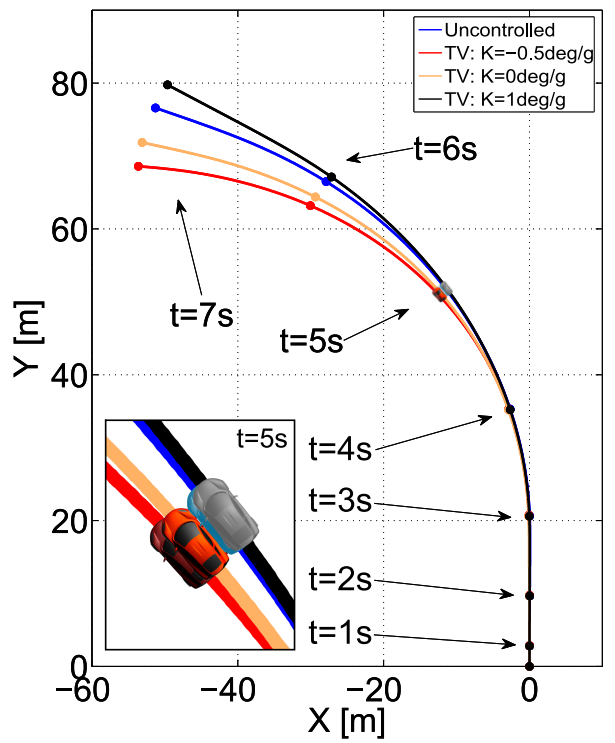

Fig. 14. Constant acceleration and step steer manoeuvre XY coordinates, comparing the trajectory of the uncontrolled car and the car with TV on, for three different understeer gradient targets

creases, the range of available yaw moments $\left(M_{z, \max }-M_{z, \min }\right)$ exceeds the minimum threshold and TV is activated at $\mathrm{t}=1.6 \mathrm{~s}$ (see Fig. 15(b)). Firstly, the vehicle has a mild oversteer with respect to target of $0 \mathrm{deg} / \mathrm{g}$, and the controller modulates front/rear torque by shifting it more towards the rear (Fig. 15(c)). From $\mathrm{t}=2.7 \mathrm{~s}$ however, the car exhibits understeer (Fig. 15(d)) and the controller starts to favour the torque balance towards the rear axle (again Fig. 15(c)). The limit lateral acceleration is reached at $\mathrm{t}=3.9 \mathrm{~s}$. The difference in trajectory between the passive and the controlled vehicle becomes significant between $\mathrm{t}=3.0 \mathrm{~s}$ and $\mathrm{t}=3.9 \mathrm{~s}$ - when the car approaches the limit of adhesion - as the passive car exhibits a non-linear yaw gain behaviour, whereas the TV controller is able to provide a linear handling characteristic to the vehicle throughout the entire manoeuvre.

Finally, the results for the constant steer manoeuvre with a target $K_{U}=1.0 \mathrm{deg} / \mathrm{g}$ are shown in Figure 16. It implies an understeer target with respect to the uncontrolled car. The TV is activated at $\mathrm{t}=1.6 \mathrm{~s}$ and its actuation is saturated sending as much torque as it can to the front axle to induce the minimum (most negative) yaw moment. However, it is not until $\mathrm{t}=3.1 \mathrm{~s}$ that the demanded yaw moment $M_{z, \text { req,ideal }}$ can be met within the range of available yaw moments $\left(M_{z, \max }-M_{z, \min }\right)$, once the lateral acceleration and tyre usage increase enough to exploit the longitudinal/lateral coupling effects. Then, the trajectory is closely followed. At $\mathrm{t}=4.4 \mathrm{~s}$, the limit lateral acceleration is reached.

For this constant steer manouvre, the effect of the two different understeer targets on the vehicle's trajectories over the uncontrolled vehicle, are visually shown in Figure 17. The yaw behaviour of the car is quite modified, although in lesser magnitude than in the step steer manoeuvres. This is due to various reasons: the manoeuvres have shorter duration compared to the step steer manoeuvres, the TV is not active dur-
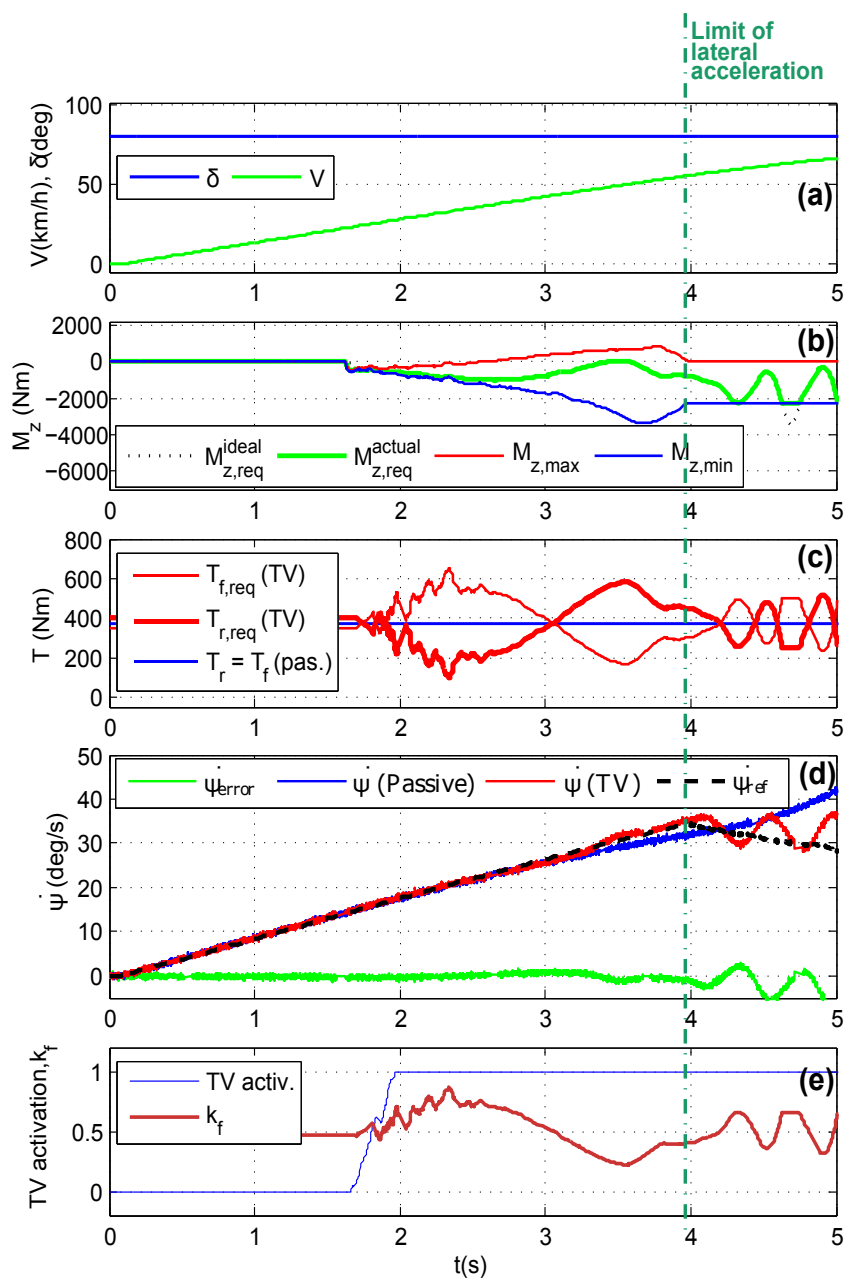

Fig. 15. Constant acceleration and constant steer manoeuvre, with an understeer target gradien $K_{U}=0.0 \mathrm{deg} / \mathrm{g}$ : (a) Steering wheel angle and vehicle speed, (b) Calculated yaw moments in the Allocation Algorithm, (c) Axle torque requests, (d) Measured yaw rates versus yaw rate reference (f) TV transitioning (activation) factor and front/rear torque distribution $k_{f}$

ing the first $\mathrm{t}=1.6 \mathrm{~s}$ and one of the understeer targets used $K_{U}=0.0 \mathrm{deg} / \mathrm{g}$ - was very close to the natural behaviour of the uncontrolled car.

\section{Conclusions}

The research presented in this paper proves the capabilities of the front/rear TV topology in actively shaping vehicle handling characteristics in battery electric vehicles with one actuator per axle. This configuration is likely to be implemented in future electric all-wheel-drive vehicles over individual active corners topologies, due to reduced cost and complexity. In particular:

- The proposed formulation of the Control Allocation in terms of high-level vehicle forces and yaw moment 

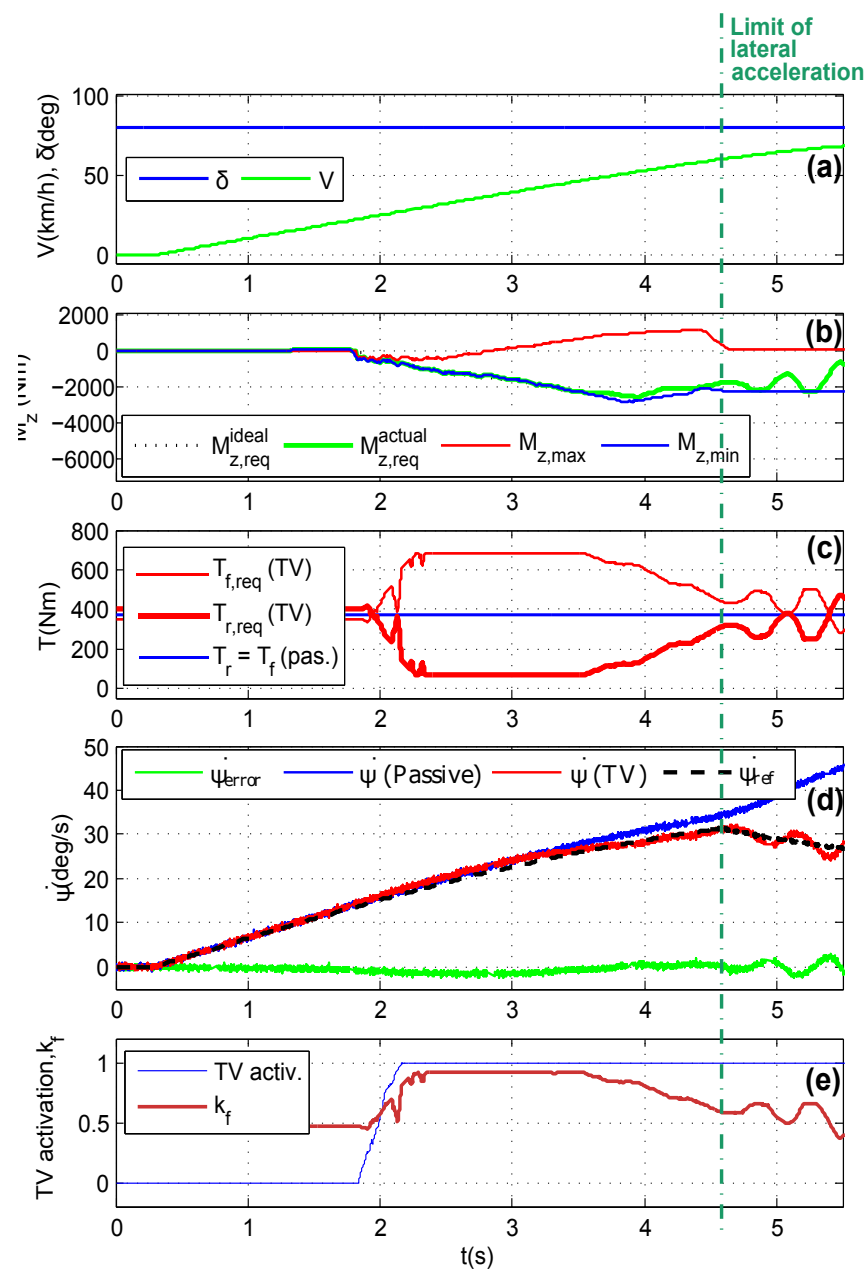

Fig. 16. Constant acceleration and constant steer manoeuvre, with an understeer target gradien $K_{U}=1.0 \mathrm{deg} / \mathrm{g}$ : (a) Steering wheel angle and vehicle speed, (b) Calculated yaw moments in the Allocation Algorithm, (c) Axle torque requests, (d) Measured yaw rates versus yaw rate reference (f) TV transitioning (activation) factor and front/rear torque distribution $k_{f}$

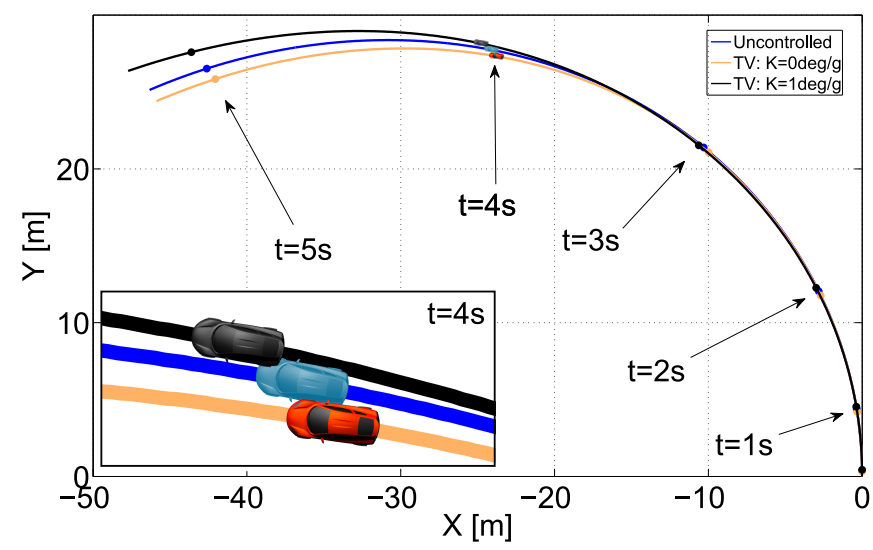

Fig. 17. Constant acceleration and constant steer manoeuvre $X Y$ coordinates, comparing the trajectory of the uncontrolled car and the car with TV on, for two different understeer gradient targets allows to consider tyre forces and motor limits straightforwardly.

- The online feasible yaw moment range computation, considering a set of measured/estimated inputs enables to evaluate at every instant if the TV capability is insufficient to alter the handling behaviour. In this case the system can be switched off in favour of a torque split strategy proportional to axle normal loads.

- A graphic representation (obtained with an offline analysis process) of the yaw moment envelope have been presented to show the influence of different front/rear torque distributions on the realisable yaw moment. This allow an a priori/offline assessment of the capabilities of the TV strategy for different longitudinal demands, and the influence of different factors on performance: i.e., maximum and minimum permissible front/rear torque distributions, tyres adhesion and motor limits.

- The front/rear TV controller was implemented on a state-of-the-art HiL rig for step steer and constant steer manoeuvres, with different targets, both understeer and oversteer. The results prove real-time capability as well as the ability to properly shape the lateral dynamics of the vehicle, especially on sub-limit conditions in presence of high lateral accelerations.

For the case of a car with a natural tendency to understeer, the proposed controller increases fun-to-drive in a predictable, controlled a safe way. Since the strategy relies on longitudinal/lateral tyre forces coupling, manoeuvres presenting high, but not excessive, sideslip and significant longitudinal acceleration have shown the best opportunity to tailor the lateral dynamics of the car to the drivers desire.

\section{Funding}

This work is undertaken within the Evoque_e project, cofunded by the UK's innovation agency, Innovate UK.

\section{References}

[1] Bartels, M., Liu, Q., Kaiser, G., and Werner, H., 2013. "Lpv torque vectoring for an electric vehicle using parameter-dependent lyapunov functions". In 2013 American Control Conference, pp. 2153-2158.

[2] De Novellis, L., Sorniotti, A., Gruber, P., Orus, J., Fortun, J. M. R., Theunissen, J., and De Smet, J., 2015. "Direct yaw moment control actuated through electric drivetrains and friction brakes: Theoretical design and experimental assessment”. Mechatronics, 26, pp. 1 15.

[3] Goggia, T., Sorniotti, A., Ferrara, A., De Novellis, L., Pennycott, A., and Gruber, P., 2015. "Integral sliding mode for the yaw moment control of four-wheel-drive fully electric vehicles with in-wheel motors". International Journal of Powertrains, 4, pp. 388-419.

[4] Q. Wang, B. A., and Singh, A., 2016. "Control allocation for multi-axle hub motor driven land vehicles". 
SAE International Journal of Alternative Powertrains 5, 2, April, pp. 338-347.

[5] Piyabongkarn, D., Lew, J. Y., Rajamani, R., Grogg, J. A., and Yuan, Q., 2007. "On the use of torque-biasing systems for electronic stability control: Limitations and possibilities". IEEE Transactions on Control Systems Technology, 15, May, pp. 581-589.

[6] He, Z., Ou, Y., and Yuan, J., 2013. "Research on the torque dynamic distribution algorithm of in-wheelmotor electric vehicle". In Proceedings of the FISITA 2012 World Automotive Congress, Springer, pp. 257266.

[7] Wheals, J. C., Baker, H., Ramsey, K., and Turner, W., 2004. Torque vectoring AWD driveline: Design, simulation, capabilities and control. SAE Technical Paper 2004-01-0863.

[8] Wheals, J., 2005. Torque vectoring driveline: SUVbased demonstrator and practical actuation technologies. SAE Technical Paper 2005-01-0553.

[9] Wheals, J., Deane, M., Drury, S., and Griffith, G., 2006. Design and simulation of a torque vectoring rear axle. SAE Technical Paper 2006-01-0818.

[10] Sill, J., and Ayalew, B., 2013. "A saturation balancing control method for enhancing dynamic vehicle stability". International Journal of Vehicle Design, 61, April, pp. 47-66.

[11] Siampis, E., Velenis, E., and Longo, S., 2016. CRC Press, Apr, ch. Front-to-rear torque vectoring Model Predictive Control for terminal understeer mitigation, pp. 153-160. 0.

[12] Soltani, A., 2014. "Low cost integration of Electric Power-Assisted Steering (EPAS) with Enhanced Stability Program (ESP)". PhD thesis, Cranfield University.

[13] Polesel, M., Shyrokau, B., Tanelli, M., Savitski, D., Ivanov, V., and Ferrara, A., 2014. "Hierarchical control of overactuated vehicles via sliding mode techniques". In Decision and Control (CDC), 2014 IEEE 53rd Annual Conference on, IEEE, pp. 4095-4100.

[14] Pennycott, A., De Novellis, L., Sorniotti, A., and Gruber, P., 2014. The Application of Control and Wheel Torque Allocation Techniques to Driving Modes for Fully Electric Vehicles. Tech. Rep. 2014-01-0085, SAE International, Warrendale, PA, Apr.

[15] Kaiser, G., Liu, Q., Hoffmann, C., Korte, M., and Werner, H., 2012. "Torque vectoring for an electric vehicle using an LPV drive controller and a torque and slip limiter". In Decision and Control (CDC), 2012 IEEE 51st Annual Conference on, IEEE, pp. 50165021.

[16] Rubin, D., and Arogeti, S. A., 2015. "Vehicle yaw stability control using active limited-slip differential via model predictive control methods". Vehicle System Dynamics, June, pp. 1-16.

[17] Tchamna, R., and Youn, I., 2013. "Yaw rate and sideslip control considering vehicle longitudinal dynamics". International Journal of Automotive Technology, 14, Feb., pp. 53-60.

[18] Siampis, E., Velenis, E., and Longo, S., 2015. "Predic- tive rear wheel torque vectoring control with terminal understeer mitigation using nonlinear estimation". In 2015 54th IEEE Conference on Decision and Control (CDC), pp. 4302-4307.

[19] MathWorks, 2015. PID Controller, Discrete PID Controller.

[20] Pacejka, H. B., 2006. Tire and vehicel dynamics. 